\title{
Normal forms and global phase portraits of quadratic and cubic integrable vector fields having two nonconcentric circles as invariant algebraic curves
}

\author{
Jaume Llibre, Marcelo Messias \& Alisson C. Reinol
}

To cite this article: Jaume Llibre, Marcelo Messias \& Alisson C. Reinol (2017) Normal forms and global phase portraits of quadratic and cubic integrable vector fields having two nonconcentric circles as invariant algebraic curves, Dynamical Systems, 32:3, 374-390, DOI: 10.1080/14689367.2016.1263600

To link to this article: https://doi.org/10.1080/14689367.2016.1263600

Accepted author version posted online: 23

Nov 2016.

Published online: 14 Dec 2016.

Submit your article to this journal $2 \pi$

Џll Article views: 84

View Crossmark data ¿ 


\title{
Normal forms and global phase portraits of quadratic and cubic integrable vector fields having two nonconcentric circles as invariant algebraic curves
}

\author{
Jaume Llibre ${ }^{a}$, Marcelo Messias ${ }^{b}$ and Alisson C. Reinol ${ }^{b}$ \\ ${ }^{a}$ Departament de Matemàtiques, Universitat Autònoma de Barcelona, Barcelona, Spain; ${ }^{\mathrm{b}}$ Departamento de \\ Matemática e Computação, Faculdade de Ciências e Tecnologia, Universidade Estadual Paulista, UNESP, \\ P. Prudente, Brazil
}

\section{ABSTRACT}

In this paper, we give the normal form of all planar polynomial vector fields of degree $d \leq 3$ having two nonconcentric circles $C_{1}$ and $C_{2}$ as invariant algebraic curves and the function $H=C_{1}^{\beta} C_{2}^{\alpha}$, with $\alpha$ and $\beta$ real values, as first integral. Moreover, we classify all global phase portraits on the Poincaré disc of a subclass of these vector fields.

\section{ARTICLE HISTORY}

Received 15 February 2014 Accepted 14 November 2016

\section{KEYWORDS}

Quadratic and cubic vector fields; invariant algebraic curves; limit cycles; Poincaré compactification; global analysis; normal forms

MCS/CCS/AMS CLASSIFICATION/CR CATEGORY NUMBERS: 34C05, 34C07, 34C08, 34C20, $34 \mathrm{C} 25,37 \mathrm{G} 10$

\section{Introduction and statement of the main result}

In this work, we deal with the following planar polynomial differential system:

$$
\dot{x}=P(x, y), \quad \dot{y}=Q(x, y),
$$

where $P$ and $Q$ are polynomials in the real variables $x$ and $y$ with degree $d=\max \{\operatorname{deg}(P)$, $\operatorname{deg}(Q)\}$, where $\operatorname{deg}(P)$ and $\operatorname{deg}(Q)$ are the degrees of $P$ and $Q$, respectively. In system (1), the dot denotes derivative with respect to the time $t$. When $d=3$, we say that system (1) is $c u b i c$, and when $d=2$, we say that it is quadratic. Here, we consider $d \leq 3$. We can associate to system (1) the vector field $X=(P, Q)$ defined on $\mathbb{R}^{2}$. Beyond its theoretical importance, systems like (1) appear frequently in many branches of applied mathematics, as for example in modelling physical and biological problems. An important question about system (1) is the determination and localization of its limit cycles, which is related to the well-known Hilbert 16th problem [4].

As the whole class of systems (1) is very difficult to be studied, even in the quadratic case, one of the strategies to study them is to consider subclasses of these systems, satisfying some 
properties, as for instance the subclasses studied in $[1,5,6]$ and references therein. In order to establish certain properties to system (1), we introduce some definitions of Darboux theory of integrability. This kind of integrability provides a link between the integrability of vector fields and their invariant algebraic curves. More details about this theory can be found in Chapter 2 of [2] and in Chapter 8 of [3]. Denote by $\mathbb{R}[x, y]$ the ring of polynomials in the real variables $x$ and $y$ and coefficients in $\mathbb{R}$ and let $f \in \mathbb{R}[x, y] \backslash \mathbb{R}$, that is, $f$ is a nonconstant polynomial. The algebraic curve $f(x, y)=0$ is an invariant algebraic curve of system (1) if for some polynomial $K \in \mathbb{R}[x, y]$ we have

$$
X(f)=P \frac{\partial f}{\partial x}+Q \frac{\partial f}{\partial y}=K f .
$$

The polynomial $K$ is called the cofactor of the invariant algebraic curve $f=0$.

In Theorem 2.1 of Section 2, we shall prove that the most general normal form of planar polynomial vector fields having two invariant algebraic curves $f_{1}=0$ and $f_{2}=0$, is

$$
X=\lambda_{1} f_{1} H_{f_{2}}+\lambda_{2} f_{2} H_{f_{1}}+f_{1} f_{2} \tilde{X}
$$

where $H_{f_{i}}$ denotes the Hamiltonian vector field $\left(-\partial f_{i} / \partial y, \partial f_{i} / \partial x\right), \lambda_{i}$ are arbitrary rational functions, for $i=1,2$, and $\tilde{X}$ is an arbitrary polynomial vector field. As an example, consider the polynomial vector field

$$
X_{0}=\left(3 y\left(3 x^{2}+2 x y+y^{2}-9 x-3 y+8\right),-6 x^{3}-3 x^{2} y+3 y^{3}+27 x^{2}+9 x y-27 x-3 y\right)
$$

of degree 3 obtained from (2) taking

$$
\begin{aligned}
f_{1}(x, y) & =x^{2}+y^{2}-1=0, \\
f_{2}(x, y) & =(x-3)^{2}+y^{2}-1=0, \\
\alpha & =-(x+y) / 2, \\
\beta & =-(\alpha+3 / 2), \\
\tilde{X} & =0 .
\end{aligned}
$$

It is not difficult to show that the cubic polynomial vector field $X_{0}$ has the circles $f_{1}=0$ and $f_{2}=0$ as limit cycles, i.e. these two circles are periodic orbits isolated in the set of all periodic orbits of the vector field (2). The phase portrait of $X_{0}$ in the Poincare disc is given in Figure 1. Note that $X_{0}$ is an example of a cubic non-integrable vector field having two nonconcentric circles as invariant algebraic curves. This kind of vector fields may have a complicated behaviour.

Since this is the first work on quadratic and cubic vector fields having two nonconcentric invariant circles $C_{1}$ and $C_{2}$, we will restrict our goal to study the integrable ones, whose first integral is constructed uniquely using the nonconcentric invariant circles, that is, the first integral is the function $H=C_{1}^{\beta} C_{2}^{\alpha}$, with $\alpha$ and $\beta$ real values. After an appropriate change of coordinates, we can suppose without loss of generality that the invariant circles $C_{1}$ and $C_{2}$ are given by

$$
C_{1}(x, y)=x^{2}+y^{2}-1=0 \quad \text { and } \quad C_{2}(x, y)=(x-a)^{2}+y^{2}-r^{2}=0,
$$

with $a>0$ and $r>0$. 




Figure 1. Phase portrait having two non-nested invariant circles which are limit cycles.

In Proposition 2.3 of Section 2, we shall prove that the normal form of all planar polynomial vector fields of degree $d \leq 3$ having the two nonconcentric invariant circles $C_{1}$ and $C_{2}$, given by (3), and a first integral given by $H=C_{1}^{\beta} C_{2}^{\alpha}$, with $\alpha$ and $\beta$ real values, is

$$
X=\lambda_{1} C_{1} H_{C_{2}}+\lambda_{2} C_{2} H_{C_{1}}
$$

where $H_{C_{i}}$ denotes the Hamiltonian vector field $\left(-\partial C_{i} / \partial y, \partial C_{i} / \partial x\right)$, for $i=1,2$, and $\lambda_{1}$ and $\lambda_{2}$ are arbitrary rational functions such that $\beta \lambda_{1}=\alpha \lambda_{2}$. Finally, we do a study of the global phase portraits of the vector fields

$$
X=\alpha C_{1} H_{C_{2}}+\beta C_{2} H_{C_{1}}
$$

obtained as a subclass of vector fields (4) taking $\lambda_{1}=\alpha$ and $\lambda_{2}=\beta$. As we shall see in Proposition 2.4, these vector fields have no limit cycles.

All the phase portraits of the vector field (5) presented in this note are drawn on the Poincaré disc. More precisely, the Poincaré compactification extends a polynomial vector field $X=(P, Q)$ to a vector field $\pi(X)$ defined on the compact manifold $\mathbb{S}^{2}$ (the unit sphere of $\mathbb{R}^{3}$ centred at the origin), giving two copies of the vector field $X$, one in the open northern hemisphere of $\mathbb{S}^{2}$ and the other on the open southern hemisphere, in such a way that the flow on the equator corresponds to the flow of the vector field $X$ at infinity. Using this technique, the global phase portraits of $X$ become completely determined whenever we consider only the phase portraits of $\pi(X)$ restricted to the northern closed hemisphere and we project it on the closed unit disc on $\mathbb{R}^{2}$, called the Poincaré disc. For more details and definition on the Poincaré disc, see Chapter 5 of [3].

Aiming to determine the global phase portraits of vector field (5), it is important to consider the possible relative positions between the circles $C_{1}$ and $C_{2}$ given in (3) (see Figure 2), since the dynamical behaviour of the solutions are directly related to their relative position.

Let $X$ and $Y$ be two polynomial vector fields of $\mathbb{R}^{2}$. We say that these vector fields are topologically equivalent if there exists a homeomorphism $h: \mathbb{R}^{2} \rightarrow \mathbb{R}^{2}$ such that $h$ carries orbits under the flow of $X$ onto orbits under the flow of $Y$, preserving or reversing the sense of these orbits.

We state below one of the main results of this paper, which gives all the possible phase portraits on the Poincaré disc of vector field (5), under topological equivalence. 




Figure 2. Possible relative positions between the circles $C_{1}$ and $C_{2}$ given in (3).

Theorem 1.1: The phase portrait on the Poincaré disc of vector field (5) is topologically equivalent to one of the 22 phase portraits described in Figure 3

This paper is devoted to prove Theorem 1.1. The phase portraits 1,2 and 3 of Figure 3 correspond to quadratic systems and all the others correspond to cubic systems.

In the rest of this paper, we present a global study of vector field (5) as follows. In Section 2, we derive the normal form (5) as a subclass of the vector field (4) and, as a consequence, we prove that vector field (5) has no limit cycles. In Section 3, we consider the quadratic vector fields (5), obtained by taking $\alpha=-\beta$, and we describe all the possible global phase portraits for this subclass. In Section 4, we do a global analysis of vector field (5), with $\alpha \neq-\beta$, considering three cases: $\alpha=0$ and $\beta \neq 0 ; \beta=0$ and $\alpha \neq 0$; and $\alpha \beta \neq 0$.

\section{Normal forms}

Let $h_{1}$ and $h_{2}$ be functions defined in an open subset $U$ of $\mathbb{R}^{2}$. We define the Jacobian matrix of $h_{1}$ and $h_{2}$ as

$$
J=\left(\begin{array}{l}
\partial h_{1} / \partial x \partial h_{1} / \partial y \\
\partial h_{2} / \partial x \partial h_{2} / \partial y
\end{array}\right)
$$

The Jacobian of $J$, i.e. the determinant of $J$, is denoted here by

$$
|J|:=\left\{h_{1}, h_{2}\right\}
$$

The next result is inspired in Theorem 5 of [7], which characterizes all vector fields hav$\operatorname{ing} f_{i}=0$, for $i=1, \ldots, n$, as invariant algebraic curves such that $\left\{f_{1}, \ldots, f_{n}\right\} \not \equiv 0$. Here, we present a shorter and direct proof for the particular case of planar polynomial vector fields having two invariant algebraic curves. 

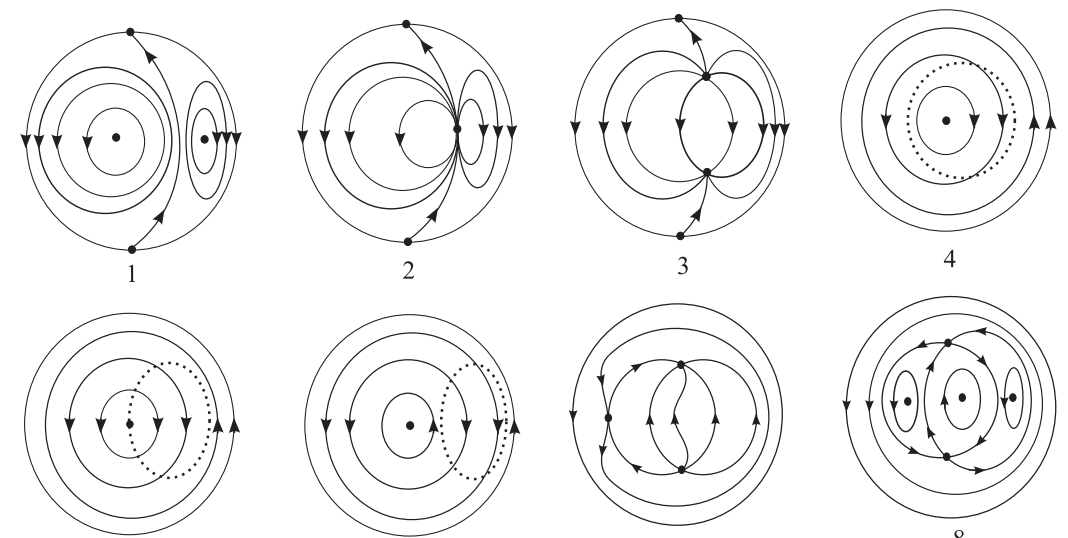

6


10

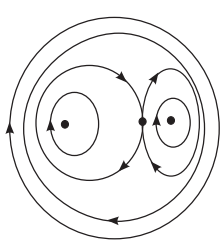

13

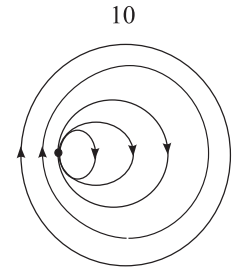

11

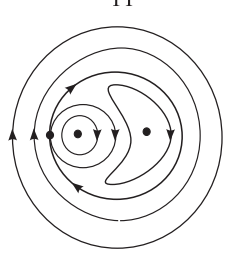

15

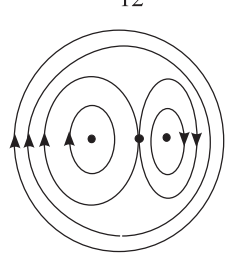

16
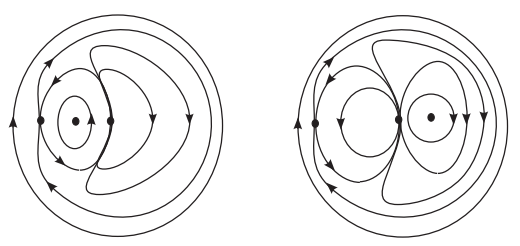

18

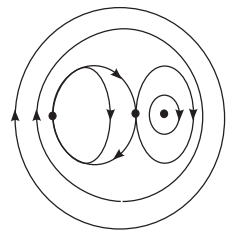

19

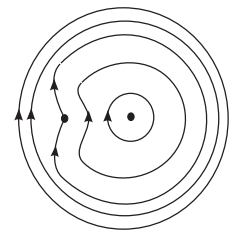

20

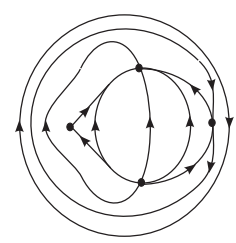

21

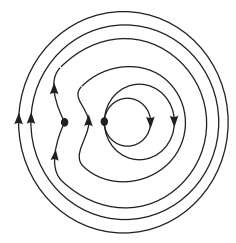

22

Figure 3. Possible phase portraits of vector field (5) on the Poincaré disc.

Theorem 2.1: Let $f_{1}$ and $f_{2}$ be irreducible polynomials on $\mathbb{R}[x, y]$ such that the Jacobian $\left\{f_{1}, f_{2}\right\} \not \equiv 0$. Then, any planar polynomial vector field which admits $f_{1}=0$ and $f_{2}=0$ as invariant algebraic curves can be written in the form (2). 
Proof: Suppose that $X=(P, Q)$ is a polynomial vector field which admits $f_{1}=0$ and $f_{2}=0$ as invariant algebraic curves. Then, by definition,

$$
\begin{aligned}
& X\left(f_{1}\right)=P \frac{\partial f_{1}}{\partial x}+Q \frac{\partial f_{1}}{\partial y}=K_{1} f_{1}, \\
& X\left(f_{2}\right)=P \frac{\partial f_{2}}{\partial x}+Q \frac{\partial f_{2}}{\partial y}=K_{2} f_{2},
\end{aligned}
$$

where $K_{1}$ and $K_{2}$ are the cofactors of $f_{1}=0$ and $f_{2}=0$, respectively. Hence, multiplying Equation (7) by $\partial f_{1} / \partial y$ and substituting $Q \partial f_{1} / \partial y$ from (6) into (7), we obtain

$$
P=\frac{-K_{1}}{\left\{f_{1}, f_{2}\right\}} f_{1}\left(-\frac{\partial f_{2}}{\partial y}\right)+\frac{K_{2}}{\left\{f_{1}, f_{2}\right\}} f_{2}\left(-\frac{\partial f_{1}}{\partial y}\right) .
$$

Now multiplying Equation (7) by $\partial f_{1} / \partial x$ and substituting $P \partial f_{1} / \partial x$ from (6) into (7), we obtain

$$
Q=\frac{-K_{1}}{\left\{f_{1}, f_{2}\right\}} f_{1} \frac{\partial f_{2}}{\partial x}+\frac{K_{2}}{\left\{f_{1}, f_{2}\right\}} f_{2} \frac{\partial f_{1}}{\partial x} .
$$

By hypothesis, $P$ and $Q$ are polynomials and $f_{1}$ and $f_{2}$ are irreducible polynomials, then, we can consider $K_{1}=-\left\{f_{1}, f_{2}\right\} \lambda_{1}$ and $K_{2}=\left\{f_{1}, f_{2}\right\} \lambda_{2}$, with $\lambda_{1}$ and $\lambda_{2}$ arbitrary rational functions. Therefore, we obtain $X=\lambda_{1} f_{1} H_{f_{2}}+\lambda_{2} f_{2} H_{f_{1}}$. Note that even $\lambda_{1}$ and $\lambda_{2}$ being rational functions, we can choose them in such a way that $X$ is a polynomial vector field.

However, this is not the most general form of vector field $X$. Indeed, since the flow of $X$ preserves $f_{1}=0$ and $f_{2}=0$, we can add $f_{1} f_{2} \tilde{X}$, where $\tilde{X}$ is an arbitrary planar polynomial vector field, into the expression of $X$. Then, the most general form of $X$ is given by (2).

Before introducing the normal form (4), we present the next result which gives us a way to construct first integrals using invariant algebraic curves and we can find a proof for it in [3] (see statement (i) of Theorem 8.7).

Proposition 2.2: Suppose that a polynomial differential system (1) of degree $d$ admits $p$ invariant algebraic curves $f_{i}=0$ with cofactors $K_{i}$, for $i=1, \ldots$, p. There exist $\mu_{i} \in \mathbb{R}$ not all zero such that

$$
\sum_{i=1}^{p} \mu_{i} K_{i}=0
$$

if and only if the function $H=f_{1}^{\mu_{1}}+\cdots+f_{p}^{\mu_{p}}$ is the first integral of $X$.

Proposition 2.3: Any planar polynomial vector field $X$ of degree $d \leq 3$ having two nonconcentric circles $C_{1}$ and $C_{2}$ as invariant algebraic curves and the function $H=C_{1}^{\beta} C_{2}^{\alpha}$, with $\alpha$ and $\beta$ real values, as first integral, after a change of coordinates can be written as (4).

Proof: After an adequate change of coordinates, we can suppose that the equations of the two nonconcentric invariant circles $C_{1}$ and $C_{2}$ are given by (3). By Theorem 2.1, we can write vector field $X$ as (2) taking $f_{i}=C_{i}$, for $i=1,2$ (remember that $a \neq 0$ so the Jacobian 
$\left.\left\{C_{1}, C_{2}\right\} \not \equiv 0\right)$. Since $H=C_{1}^{\beta} C_{2}^{\alpha}$, with $\alpha$ and $\beta$ real values, is the first integral of $X$, from Proposition 2.2, we obtain the equation

$$
\beta K_{1}+\alpha K_{2}=0
$$

where $K_{1}=-4 a y \lambda_{1}+2 C_{2}(x \tilde{P}+y \tilde{Q})$ and $K_{2}=4 a y \lambda_{2}+2 C_{1}((x-a) \tilde{P}+y \tilde{Q})$, with $\tilde{X}=$ $(\tilde{P}, \tilde{Q})$ into $(2)$, are the cofactors of $C_{1}=0$ and $C_{2}=0$, respectively. From (8), we obtain that $\tilde{P} \equiv \tilde{Q} \equiv 0$ and $\beta \lambda_{1}=\alpha \lambda_{2}$. Therefore, $X$ can be written as (4).

Proposition 2.3 gives the normal form of all planar polynomial vector fields of degree $d \leq 3$ having two nonconcentric circles $C_{1}$ and $C_{2}$ as invariant algebraic curves and the function $H=C_{1}^{\beta} C_{2}^{\alpha}$, with $\alpha$ and $\beta$ real values, as first integral. Now, we do a study of the global phase portraits of the vector field (4) when $\lambda_{1}=\alpha$ and $\lambda_{2}=\beta$, that is, the subclass (5). Our first result states that the vector field (5) has no limit cycles.

Proposition 2.4: The vector field (5) has no limit cycles.

Proof: We have that $H=C_{1}^{\beta} C_{2}^{\alpha}$ is the first integral of the vector field (5). More precisely, note that the function $C_{1}^{\beta-1} C_{2}^{\alpha-1}$ is an integrating factor of the vector field (5), and that this vector field multiplied by this integrating factor becomes a Hamiltonian system with Hamiltonian $H=C_{1}^{\beta} C_{2}^{\alpha}$. Since Hamiltonian vector fields cannot have limit cycles, the proposition follows.

\section{Quadratic case}

In this section, we characterize all the phase portraits on the Poincare disc of vector field (5) with degree 2 , that is, the quadratic ones. These vector fields appear when we consider $\alpha=-\beta$ into (5).

Proposition 3.1: If vector field (5) is quadratic, that is $d=2$, then it can be written as

$$
X=-C_{1} H_{C_{2}}+C_{2} H_{C_{1}}
$$

where $C_{i}=C_{i}(x, y)$ are given by (3) and $H_{C_{i}}$ denotes the Hamiltonian vector field $\left(-\partial C_{i} / \partial y\right.$, $\left.\partial C_{i} / \partial x\right)$, for $i=1,2$.

Proof: Considering an adequate change of coordinates, we can suppose that the equations of the two nonconcentric invariant circles are given by (3). Taking $\alpha=-\beta$ into vector field (5), it becomes quadratic, otherwise it is cubic, and after the rescaling of time $T=\beta t$, where $T$ is the new time, we obtain vector field (9).

Note that the function $H=C_{1} / C_{2}$ is the first integral of the vector field (9). Therefore, its phase portraits are arcs of circles. Indeed, $H=c$ is equivalent to $C_{1}-c C_{2}=0$, which is the equation of a circle. This shows that the bifurcation diagram of the vector field (9) (Figure 5) depends on whether the two circles $C_{1}$ and $C_{2}$ intersect or not: if they do, the phase portraits are circles passing through the intersection point; if they do not, the plane is foliated by such circles (Figure 6). A precise statement and an analytic proof for this case is given in the following proposition. 


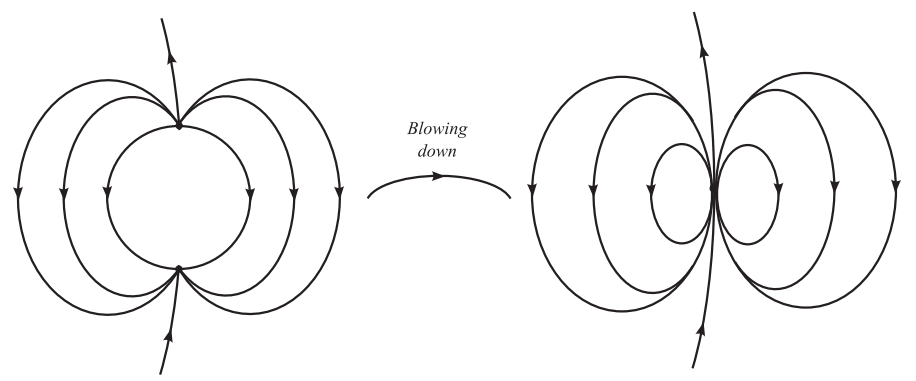

Figure 4. Polar blow-up of the singular point $R_{1}$.

Proposition 3.2: Let $A=A(a, r)=(a+1-r)(a+1+r)(a-1-r)(a-1+r)$. The following statements hold for the vector field (9):

(i) If $A<0$, then vector field (9) has two singular points, which are an unstable and a stable node, symmetric with respect to the $x$-axis. Moreover, these singular points belong to the intersection of the invariant circles $C_{1}$ and $C_{2}$.

(ii) If $A=0$, then vector field (9) has only one singular point, which is on the $x$-axis and it is a nonelementary singular point, formed by the union of two elliptic sectors (see Figure 4). Moreover, it belongs to the tangency point between the invariant circles $C_{1}$ and $\mathrm{C}_{2}$.

(iii) If $A>0$, then vector field (9) has two singular points, which are centres belonging to the $x$-axis.

Proof: Computing the singular points of vector field (9), we obtain

$$
\begin{array}{ll}
M_{1}=\left(\frac{1+a^{2}-r^{2}}{2 a}, \frac{\sqrt{-A}}{2 a}\right), & N_{1}=\left(\frac{1+a^{2}-r^{2}}{2 a},-\frac{\sqrt{-A}}{2 a}\right), \\
P_{1}=\left(\frac{1+a^{2}-r^{2}+\sqrt{A}}{2 a}, 0\right), & Q_{1}=\left(\frac{1+a^{2}-r^{2}-\sqrt{A}}{2 a}, 0\right),
\end{array}
$$

where $A=A(a, r)=(a+1-r)(a+1+r)(a-1-r)(a-1+r)$. Therefore, we have three cases to consider: $A<0, A=0$ and $A>0$.

(i) If $A<0$, then from (10), only $M_{1}$ and $N_{1}$ are singular points of vector field (9). Note that $M_{1}$ and $N_{1}$ are symmetric with respect to the $x$-axis. The eigenvalues of the Jacobian matrix of vector field (9) at the point $M_{1}$ are $\lambda_{1}=\lambda_{2}=\sqrt{-A}$, while the eigenvalues at the point $N_{1}$ are $\lambda_{1}=\lambda_{2}=-\sqrt{-A}$. Thus, $M_{1}$ is an unstable node and $N_{1}$ is a stable node. Furthermore, taking $x=\left(1+a^{2}-r^{2}\right) /(2 a)$ and $y=\sqrt{-A} /(2 a)$, i.e. $x$ and $y$ are given by the coordinates of $M_{1}$, then $x$ and $y$ satisfy simultaneously the equations $x^{2}+y^{2}-1=0$ and $(x-a)^{2}+y^{2}-r^{2}=0$. Hence, $M_{1} \in C_{1} \cap C_{2}$. Analogously, we obtain that $N_{1} \in C_{1} \cap C_{2}$.

(ii) If $A=0$, then from (10), the unique singular point of vector field (9) is

$$
R_{1}=\left(\frac{1+a^{2}-r^{2}}{2 a}, 0\right)
$$


which is on the $x$-axis. The Jacobian matrix of vector field (9) at the point $R_{1}$ is the null matrix. Hence, $R_{1}$ is a nonelementary singular point. In order to study this singularity, we use a polar blowing-up, considering the change of coordinates

$$
x=\rho \cos \theta+\frac{1+a^{2}-r^{2}}{2 a}, \quad y=\rho \sin \theta
$$

into vector field (9) and doing the rescaling $T=\rho t$, where $T$ is the new time, from which we obtain

$$
\dot{\rho}=\rho a \sin \theta, \quad \dot{\theta}=-a \cos \theta
$$

The zeros of system (11) on $\{\rho=0\}$ are located at $\theta=\pi / 2, \theta=3 \pi / 2$. It is easy to verify that $\theta=\pi / 2$ is an unstable node and $\theta=3 \pi / 2$ is a stable node. Finally, doing a blowingdown, we obtain the behaviour of solutions near the singular point $R_{1}$. Figure 4 illustrates this process. Moreover, $R_{1}$ belongs to the intersection of the invariant circles $C_{1}$ and $C_{2}$. Indeed, if we take $x=\left(1+a^{2}-r^{2}\right) /(2 a)$ and $y=0$, then $x^{2}+y^{2}-1=0$ and $(x-a)^{2}+$ $y^{2}-r^{2}=0$.

(iii) If $A>0$, then from (10), only $P_{1}$ and $Q_{1}$ are singular points of vector field (9). Note that $P_{1}$ and $Q_{1}$ are on the $x$-axis. The eigenvalues of the Jacobian matrix of vector field (9) at $P_{1}$ are $\lambda_{1,2}= \pm i \sqrt{A}$, and the eigenvalues at the point $Q_{1}$ are also $\lambda_{1,2}= \pm i \sqrt{A}$. Hence, these points can be centres or weak foci of vector field (9). As the function

$$
H=\frac{(x-a)^{2}+y^{2}-r^{2}}{x^{2}+y^{2}-1}
$$

is the first integral of vector field (9), so it is integrable, hence we can conclude that $P_{1}$ and $Q_{1}$ are centres.

Note that the symmetry $y \rightarrow-y$ with respect to the $x$-axis reverses the time of the flow of vector field (5); this, in particular, explains why in Proposition 3.2 the nodes are symmetric with respect to the $x$-axis and they are of opposite types. The same remark can be done for Proposition 4.2.

Proposition 3.3: The vector field (9) has two infinite singular points, which are saddles.

Proof: In order to study the infinite singular points of vector field (9), we consider the change of coordinates

$$
x=\frac{1}{\rho} \cos \theta, \quad y=\frac{1}{\rho} \sin \theta,
$$

and doing the rescaling $T=(1 / \rho) t$, where $T$ is the new time, we obtain the system

$$
\dot{\rho}=f_{0}(\theta) \rho+f_{1}(\theta) \rho^{3}, \quad \dot{\theta}=g_{0}(\theta)+g_{1}(\theta) \rho+g_{2}(\theta) \rho^{2},
$$

where $f_{i}(i=0,1)$ and $g_{j}(j=0,1,2)$ are polynomials in $\cos \theta$ and $\sin \theta$. The singular points at infinity are given by $\rho=0$ and $g_{0}(\theta)=0$, where $g_{0}(\theta)=a \cos \theta$, hence we obtain the pair of singular points $\theta=\pi / 2$ and $\theta=3 \pi / 2$. The eigenvalues of the Jacobian matrix of 


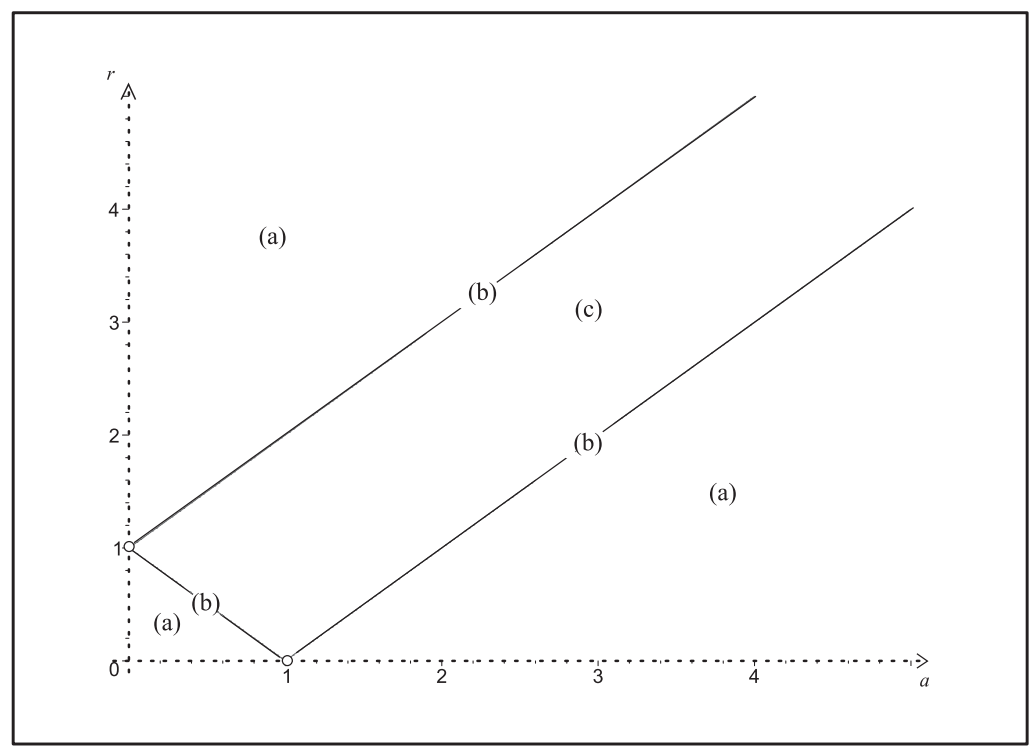

Figure 5. Bifurcation diagram of vector field (9).

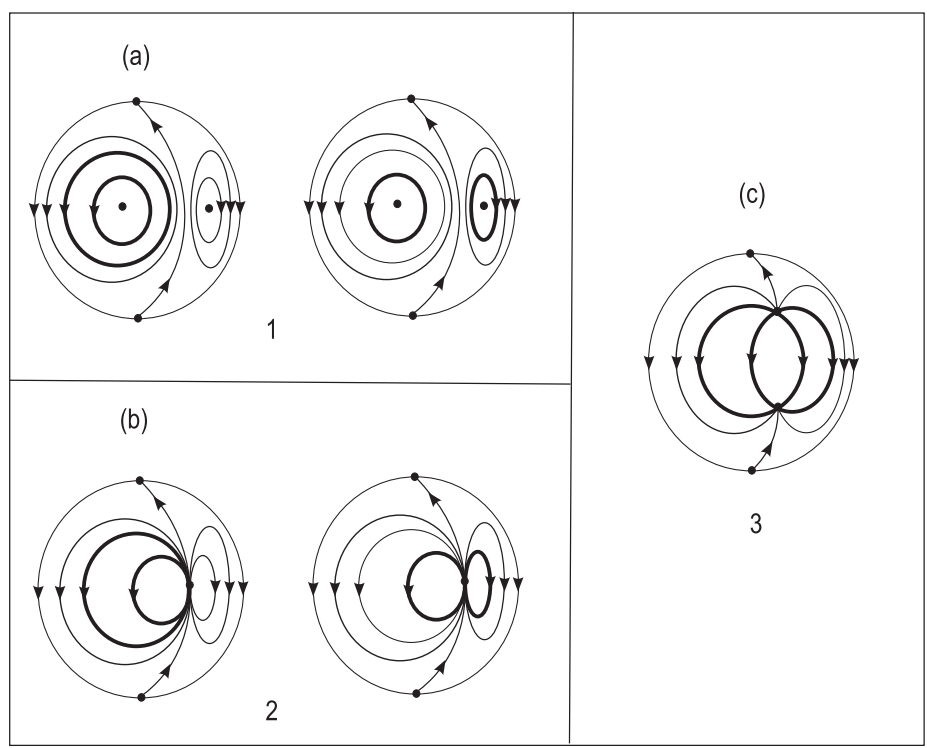

Figure 6. Phase portraits of vector field (9) on the Poincaré disc related to each region described in Figure 5, corresponding to the phase portraits 1,2 and 3 of Figure 3 . The bold curves represent the circles $C_{1}=0$ and $C_{2}=0$.

system (13) at $\theta=\pi / 2$ and at $\theta=3 \pi / 2$ are $\lambda_{1,2}= \pm a$. Thus, these infinite singular points are saddles.

The results obtained about vector field (9) are summarized in Figures 5 and 6, which show, respectively, the bifurcation diagram and the phase portraits on the Poincaré disc related to each region of the bifurcation diagram. In short, this proves Theorem 1.1 when $d=2$ providing in these cases the phase portraits 1, 2 and 3 of Figure 3. 


\section{Cubic case}

In this section, we consider the case in which the vector field (5) has degree 3 , that is, the cubic case. We shall consider three cases: case (i) $\alpha=0$ and $\beta \neq 0$; case (ii) $\beta=0$ and $\alpha \neq 0$; and case (iii) $\alpha \beta \neq 0$ and $\alpha \neq-\beta$, since the case $\alpha=-\beta$ was studied in Section 3.

\subsection{Case (i)}

Let $\alpha=0$ and $\beta \neq 0$ into (5). After the rescaling of time $T=\beta t$, where $T$ is the new time, vector field (5) reduces to

$$
X=C_{2} H_{C_{1}}
$$

which has the origin as an isolated singular point and the invariant circle $C_{2}$ is filled by singular points. Note that vector field (14) is obtained from the Hamiltonian vector field $H_{C_{1}}=(-2 y, 2 x)$ by 'slowing down' the multiplication by $C_{2}$, hence its trajectories are the same as those of $H_{C_{1}}$ or their pieces, which are cut by $\left\{C_{2}=0\right\}$. The phase portrait of vector field (14) on the Poincaré disc is one of those shown in Figure 7, providing the phase portraits 4, 5 and 6 of Figure 3. Therefore, this part of Theorem 1.1 is proved.

\subsection{Case (ii)}

Let $\beta=0$ and $\alpha \neq 0$ into (5). After the rescaling of time $T=\alpha t$, where $T$ is the new time, vector field (5) reduces to

$$
X=C_{1} H_{C_{2}},
$$

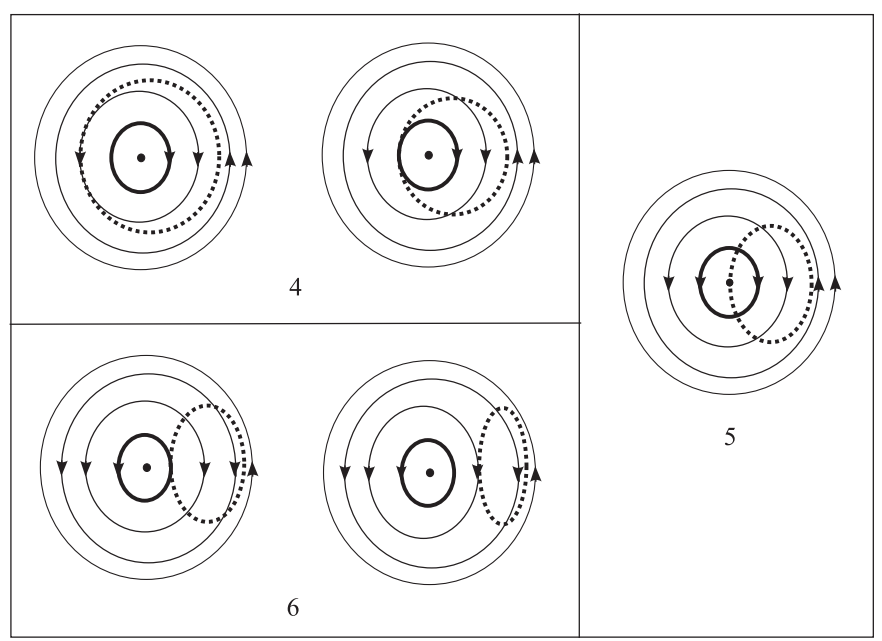

Figure 7. Phase portraits of vector field (14) on the Poincaré disc. The dotted curve represents the circle $C_{2}$, which is filled by singular points. 
which has the point $(a, 0)$ as an isolated singular point and the invariant circle $C_{1}$ is filled by singular points. Then, the phase portrait of vector field (15) on the Poincare disc is topologically equivalent to one of those shown in Figure 7, changing the dotted curve with the bold one.

\subsection{Case (iii)}

Let $\alpha \beta \neq 0$ and $\alpha \neq-\beta$ into (5). After the rescaling of time $T=\alpha t$, where $T$ is the new time, vector field (5) can be written as

$$
X=C_{1} H_{C_{2}}+\gamma C_{2} H_{C_{1}}
$$

where $\gamma=\beta / \alpha$ is a real parameter, with $\gamma \neq 0$ and $\gamma \neq-1$. In what follows, we state the following results about vector field (16): Proposition 4.1 ensures that vector field (16) has at least one and at most five singular points; Propositions 4.2 and 4.3 provide a classification for these singular points; Proposition 4.4 ensures that vector field (16) has no infinite singular points.

Proposition 4.1: Vector field (16) has at least one and at most five singular points. At least one and at most three of them are on the $x$-axis and the other two, when they exist, are symmetric with respect to the $x$-axis.

Proof: From vector field (16), we obtain the differential system

$$
\begin{aligned}
& \dot{x}=-2 y\left(C_{1}+\gamma C_{2}\right), \\
& \dot{y}=2 x\left(C_{1}+\gamma C_{2}\right)-2 a C_{1} .
\end{aligned}
$$

Note that $\dot{x}=-y f(x, y)$. So, if $\dot{x}=0$, then either $y=0$ or $f(x, y)=0$.

If we consider $f(x, y)=0$, then system (17) has the singular points

$$
M_{2}=\left(\frac{a^{2}-r^{2}+1}{2 a}, \frac{\sqrt{-A}}{2 a}\right), \quad N_{2}=\left(\frac{a^{2}-r^{2}+1}{2 a},-\frac{\sqrt{-A}}{2 a}\right),
$$

where $A=A(a, r)=(a+1-r)(a+1+r)(a-1-r)(a-1+r)$. Note that $M_{2}$ and $N_{2}$ are symmetric with respect to the $x$-axis. We must consider three cases:

(a) if $A<0$, then $M_{2}$ and $N_{2}$ are distinct singular points;

(b) if $A=0$, then $M_{2}$ and $N_{2}$ are coincident singular points;

(c) if $A>0$, then $M_{2}$ and $N_{2}$ are not singular points.

If we take $y=0$ into system (17), as

$$
\dot{y}=Q(x, y)=x\left[x^{2}+y^{2}-1+\gamma\left((x-a)^{2}+y^{2}-r^{2}\right)\right]-a\left(x^{2}+y^{2}-1\right),
$$

then,

$$
Q(x, 0)=Q(x)=(1+\gamma) x^{3}-a(2 \gamma+1) x^{2}+\left(\gamma\left(a^{2}-r^{2}\right)-1\right) x+a .
$$


Doing $Q(x)=0$, we get a third-degree equation, whose discriminant is given by the function

$$
\begin{aligned}
D=D(a, r, \gamma)= & \frac{1}{4}\left(\frac{a}{1+\gamma}+\frac{a(2 \gamma+1)\left(\gamma\left(a^{2}-r^{2}\right)-1\right)}{3(1+\gamma)^{2}}-\frac{2 a^{3}(2 \gamma+1)^{3}}{27\left(1+\gamma^{3}\right)}\right)^{2} \\
& +\frac{1}{27}\left(\frac{\gamma\left(a^{2}-r^{2}\right)-1}{1+\gamma}-\frac{a^{2}(2 \gamma+1)^{2}}{3(1+\gamma)^{2}}\right)^{3} .
\end{aligned}
$$

Therefore, we must consider three cases:

(d) if $D<0$, then equation $Q(x)=0$ has three real roots, and hence system (17) has three singular points on the $x$-axis;

(e) if $D=0$, then equation $Q(x)=0$ has two real roots, and hence system (17) has two singular points on the $x$-axis;

(f) if $D>0$, then equation $Q(x)=0$ has one real root, and hence system (17) has only one singular point on the $x$-axis.

In the next result, we give a classification for the singular points of vector field (16) symmetric with respect to the $x$-axis, that is, the singular points $M_{2}$ and $N_{2}$ given in (18). For the definitions of hyperbolic and semi-hyperbolic singular points, see, for instance, Chapter 2 of [3].

Proposition 4.2: Let $A=A(a, r)=(a+1-r)(a+1+r)(a-1-r)(a-1+r)$. The following statements hold for vector field (16).

(i) If $A<0$, then the singular points $M_{2}$ and $N_{2}$ are both saddle points or are nodes, in this last case, one is stable and the other is unstable.

(ii) If $A=0$, then $M_{2}$ and $N_{2}$ are coincident and it is a nonelementary singular point, formed by the union of two elliptic sectors, as shown in Figure 4.

(iii) If $A>0$, then $M_{2}$ and $N_{2}$ are not singular points.

Moreover, when $M_{2}$ and $N_{2}$ are singular points, they are on the intersection of the invariant circles $C_{1}$ and $C_{2}$.

Proof: According to the proof of Proposition 4.1, we have that the singular points of vector field (16) $M_{2}$ and $N_{2}$ are given by (18). Calculating the Jacobian matrix of vector field (16) at $M_{2}$, we obtain that its eigenvalues are $\lambda_{1}=-\sqrt{-A}$ and $\lambda_{2}=\gamma \sqrt{-A}$. Analogously, calculating the Jacobian matrix of vector field (16) at $N_{2}$, we have that its eigenvalues are $\lambda_{1}=\sqrt{-A}$ and $\lambda_{2}=-\gamma \sqrt{-A}$.

(i) If $A<0$, then $M_{2}$ and $N_{2}$ are distinct singular points, and in this case, we have that $\lambda_{1}$ and $\lambda_{2}$ are real eigenvalues. Hence, if $\gamma<0$, then $M_{2}$ is a stable node and $N_{2}$ is an unstable node; while for $\gamma>0, M_{2}$ and $N_{2}$ are both saddles. 




Figure 8. Nonelementary singular points of vector field (16).

(ii) If $A=0$, then $M_{2}$ and $N_{2}$ are coincident singular points and $\lambda_{1}=\lambda_{2}=0$. Therefore, $M_{2}$ and $N_{2}$ reduce to a nonelementary singular point, which is given by

$$
R_{1}=\left(\frac{1+a^{2}-r^{2}}{2 a}, 0\right) .
$$

Note that $R_{1}$ is the same singular point of the proof of item (ii) of Proposition 3.2, hence with a polar blow-up, we show that $R_{1}$ is formed by the union of two elliptic sectors, as in the proof of Proposition 3.2 (see Figure 4).

(iii) If $A>0$, then by Proposition 4.1, $M_{2}$ and $N_{2}$ are not singular points.

Finally, if we consider $x$ and $y$ given by the coordinates of $M_{2}$ and $N_{2}$, that is, $x=$ $\left(a^{2}-r^{2}+1\right) /(2 a)$ and $y= \pm \sqrt{-A} /(2 a)$, we have that they satisfy simultaneously the equations $x^{2}+y^{2}-1=0$ and $(x-a)^{2}+y^{2}-r^{2}=0$. Hence, $M_{2}, N_{2} \in C_{1} \cap C_{2}$.

Proposition 4.3: The singular points of vector field (16) on the $x$-axis are saddles, centres or nonelementary singular points, whose local phase portraits are described in Figure 8.

Proof: By Proposition 4.1, we know that vector field (16) has at least one and at most three singular points on the $x$-axis. If we consider the change of coordinates $x=\rho \cos \theta, y=$ $\rho \sin \theta$ into (16), then we obtain the system

$$
\begin{aligned}
& \dot{\rho}=-a\left(\rho^{2}-1\right) \sin \theta, \\
& \dot{\theta}=(1+\gamma) \rho^{2}+a \rho(2 \gamma-1) \cos \theta+\gamma\left(a^{2}-r^{2}\right)+1+\frac{1}{\rho} \cos \theta .
\end{aligned}
$$

In order to study the behaviour of the solutions near the singular points of vector field (16) on the $x$-axis, we calculate the Jacobian matrix of system (19) and take $\theta=0$, which have the eigenvalues $\lambda_{1,2}= \pm \sqrt{B} / \rho$, where $B=B(a, \gamma, \rho)=a\left(\rho^{2}-1\right)\left(-2(1+\gamma) \rho^{3}+a(2 \gamma\right.$ $\left.+1) \rho^{2}+a\right)$. Therefore, we have the following cases to consider.

If $B<0$, then the singular point on the $x$-axis can be a centre or a weak focus. As the function $H(x, y)=C_{1}^{\gamma} C_{2}$ is the first integral of vector field (16), then it is integrable and these singular points are centres. 




Figure 9. Phase portraits of vector field (16) on the Poincaré disc having only elementary singular points.

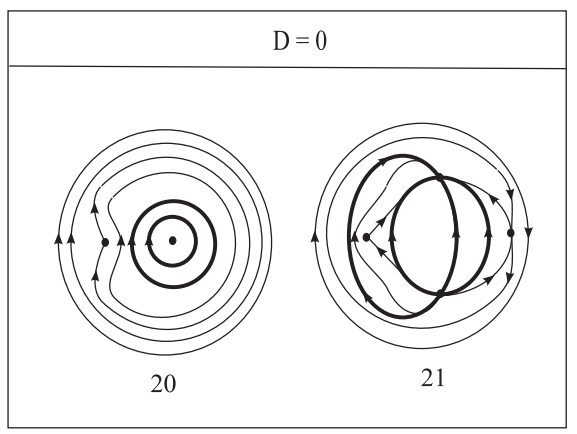

Figure 10. Phase portraits of vector field (16) on the Poincaré disc having nonelementary singular points $(D=0$ and $A \neq 0)$.

If $B=0$, then the singular point on the $x$-axis is a nonelementary singular point, since the eigenvalues are both zero. After the desingularization of this singular point using the tools of Chapters 2 and 3 of [3], we have that its local phase portrait is topologically equivalent to one of those described in Figure 8. 


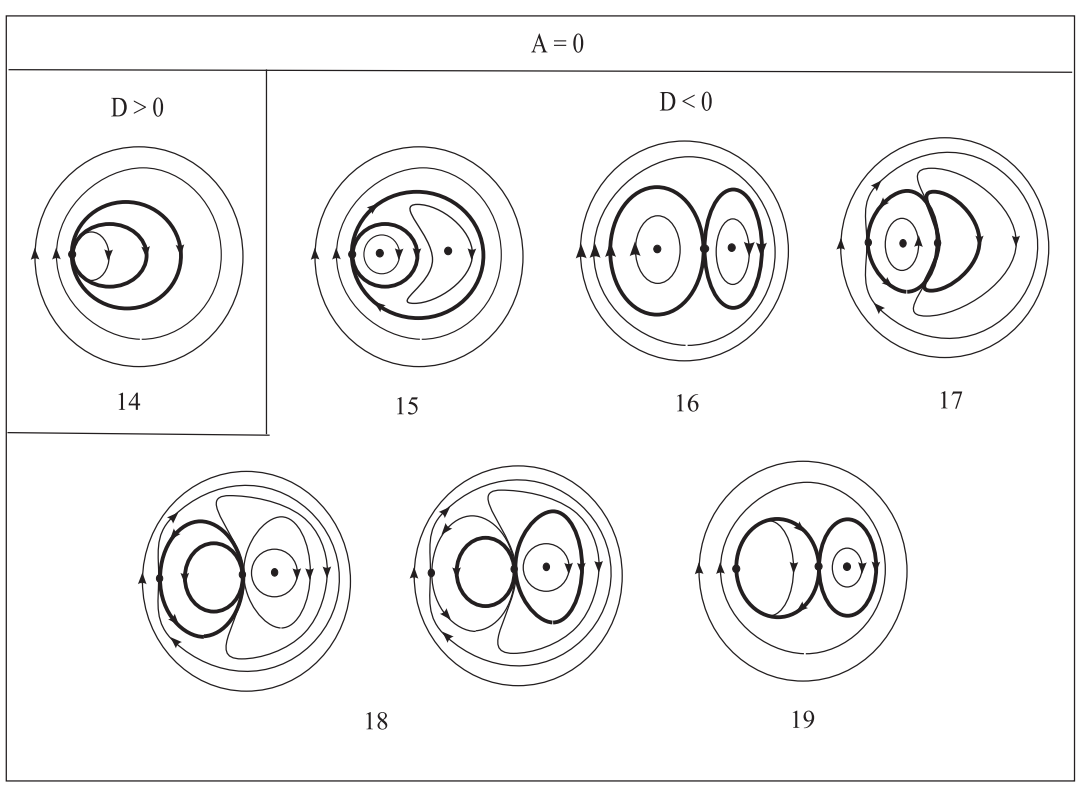

Figure 11. Phase portraits of vector field (16) on the Poincaré disc having nonelementary singular points $(A=0$ and $D \neq 0)$.

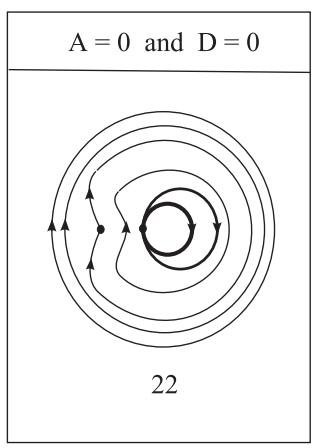

Figure 12. Phase portraits of vector field (16) on the Poincaré disc having nonelementary singular points $(A=0$ and $D=0)$.

If $B>0$, then the singular point on the $x$-axis is a saddle.

Therefore, the singular points of vector field (16) on the $x$-axis are saddles, centres or nonelementary singular points (see Figure 8), depending on the value of $B$.

Proposition 4.4: The vector field (16) has no infinite singular points.

Proof: In order to study the infinite singular points of vector field (16), we consider the change of coordinates (12) and the rescaling of time $T=\left(1 / \rho^{2}\right) t$, where $T$ is the new time, from what we obtain the system

$$
\dot{\rho}=f_{0}(\theta) \rho^{2}+f_{1}(\theta) \rho^{4}, \quad \dot{\theta}=g_{0}(\theta)+g_{1}(\theta) \rho+g_{2}(\theta) \rho^{2}+g_{3}(\theta) \rho^{3} .
$$


The infinite singular points are given by $\rho=0$ and $g_{0}(\theta)=0$. Since $g_{0}(\theta)=1$, for all $\theta \in \mathbb{R}$, then vector field (16) has no infinite singular points.

We can summarize the results about Case (iii) and classify the phase portraits of vector field (16) as follows. First, we present in Figure 9 the cases in which vector field (16) has only elementary singular points, which correspond to the phase portraits 7-13 of Figure 3. Then, we present in Figures 10-12 the other cases, when vector field (16) has nonelementary singular points, which correspond to the phase portraits 14-22 of Figure 3. In short, Figures 912 provide the phase portraits 7-22 of Figure 3, which end the proof of Theorem 1.1.

\section{Acknowledgments}

The authors thank the referee for his good comments, which helped improve the results presented in this paper.

\section{Disclosure statement}

No potential conflict of interest was reported by the authors.

\section{Funding}

The first author is partially supported by a MINECO/FEDER grant [grant number MTM200803437], [grant number MTM2013-40998-P]; an AGAUR grant [grant number 2013SGR-568]; an ICREA Academia [grant number FP7-PEOPLE-2012-IRSES 318999], [grant number 316338], [grant number FEDER-UNAB10-4E-378]; and a CAPES grant [grant number 88881.030454/201301] from the program CSF-PVE. The second author is supported by CNPq-Brazil Project [308159/2015-2] and by FAPESP Project [2013/24541-0]. The third author is supported by FAPESP Projects [2013/26602-7], [2016/01258-0].

\section{References}

[1] M. Caubergh, J. Llibre, and J. Torregrosa, Global classification of a class of cubic vector fields whose canonical regions are period annuli, Int. J. Bifur. Chaos Appl. Sci. Eng. 21 (2011), pp. 1831-1867.

[2] C. Christopher and C. Li, Limit Cycles of Differential Equations, Birkhauser-Verlag, Basel, 2007.

[3] F. Dumortier, J. Llibre, and J.C. Artés, Qualitative Theory of Planar Differential Systems, SpringerVerlag, New York, 2006.

[4] Y. Ilyashenko, Centennial history of Hilbert's 16th problem, Bull. AMS 39 (2002), pp. 301-354.

[5] J. Llibre, J.S.P. Del Río, and J.A. Rodrígues, Phase portraits of a new class of integrable quadratic vector fields, Dyn. Cont. Disc. Imp. Syst. 7 (2000), pp. 595-616.

[6] J. Llibre, A. Mahdi, and J. Roé, The geometry of the real planar polynomial differential systems having their orbits imbedded in conics, Dyn. Syst. Int. J. 26 (2011), pp. 287-321.

[7] J. Llibre, R. Ramírez, and N. Sadovskaia, Inverse approach in ordinary differential equations: Applications to Lagrangian and Hamiltonian mechanics, J. Dyn. Differ. Equat. 26 (2014), pp. 529581. 